\title{
Searching for Mg II absorbers in and around galaxy clusters
}

\author{
Jong Chul Lee ${ }^{1 \star}$, Ho Seong Hwang ${ }^{1,2} \dagger$ and Hyunmi Song ${ }^{3} \ddagger$ \\ ${ }^{1}$ Korea Astronomy and Space Science Institute, 776 Daedeokdae-ro, Yuseong-gu, Daejeon 34055, Korea \\ ${ }^{2}$ Astronomy Program, Department of Physics and Astronomy, Seoul National University, 1 Gwanak-ro, Gwanak-gu, Seoul 08826, Korea \\ ${ }^{3}$ Department of Astronomy, Yonsei University, 50 Yonsei-ro, Seodaemun-gu, Seoul 03722, Korea
}

Accepted XXX. Received YYY; in original form ZZZ

\begin{abstract}
To study environmental effects on the circumgalactic medium (CGM), we use the samples of redMaPPer galaxy clusters, background quasars and cluster galaxies from the SDSS. With $\sim 2000$ quasar spectra, we detect $197 \mathrm{Mg}$ II absorbers in and around the clusters. The detection rate per quasar is $2.7 \pm 0.7$ times higher inside the clusters than outside the clusters, indicating that $\mathrm{Mg}$ II absorbers are relatively abundant in clusters. However, when considering the galaxy number density, the absorber-togalaxy ratio is rather low inside the clusters. If we assume that $\mathrm{Mg}$ II absorbers are mainly contributed by the CGM of massive star-forming galaxies, a typical halo size of cluster galaxies is smaller than that of field galaxies by $30 \pm 10$ per cent. This finding supports that galaxy haloes can be truncated by interaction with the host cluster.
\end{abstract}

Key words: galaxies: clusters: general - galaxies: intergalactic medium - galaxies: ISM - galaxies: general - galaxies: quasars: general

\section{INTRODUCTION}

The circumgalactic medium (CGM) is a gas reservoir surrounding galaxies within their dark matter haloes. Recent cosmological simulations and observations support that the CGM is closely connected to the evolution of galaxy via gas flows (see Tumlinson et al. 2017, for a review). In the sense that the CGM harbours metal-enriched gas ejected from the galaxy, driven by star formation feedback, and fresh gas accreted from the intergalactic medium (IGM) to fuel star formation, the CGM provides an excellent laboratory for better understanding the mechanisms that control galaxy evolution.

It is challenging to detect the emission from CGM with currently available facilities because of the low surface brightness, although there are several successful attempts from deep imaging and spectroscopic observations (e.g. Cantalupo et al. 2014; Zhang et al. 2016; Daddi et al. 2020). Therefore, Ly $\alpha$ and metal absorption lines imprinted in background source spectra have been widely used to trace the CGM (e.g. Adelberger et al. 2005; Steidel et al. 2010; Prochaska et al. 2013). For the background source, quasars are popular because they are luminous objects with a simple spectral shape, generally characterized by a power-law continuum and broad emission lines, enabling us to detect foreground absorbers with a high signal-tonoise ratio $(\mathrm{S} / \mathrm{N})$. The $\operatorname{Ly} \alpha$ absorption line is very sensitive to the existence of CGM, but does not allow us to construct a large sample of Ly $\alpha$ absorbers due to observational limitations (but see Lehner et al. 2018). Among the metal absorption lines, the $\mathrm{Mg}_{\text {II }} \lambda \lambda 2796$, 2803 doublet is particularly useful for statistical studies because it is a prominent feature, visible from the ground, spanning a wide

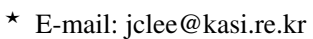

$\dagger$ E-mail: hhwang@astro.snu.ac.kr

† E-mail: hmsong@yonsei.ac.kr
}

redshift range $(0.3<z<2.5)$ in the optical wavelengths. The $\mathrm{Mg}$ II absorption is known to probe gas at temperature $\sim 10^{4} \mathrm{~K}$ (Charlton et al. 2003) with the neutral hydrogen column density $\approx 10^{16}-10^{22}$ $\mathrm{cm}^{-2}$ (Rao, Turnshek, \& Nestor 2006).

Since the first discovery of an intervening $\mathrm{Mg}$ II absorption from quasar-galaxy pairs by Bergeron (1986), the relation between galaxies and their CGM has been investigated over the last three decades. The strength of $\mathrm{Mg}$ II absorption at fixed impact parameter is found to be correlated with galaxy properties such as luminosity, mass, color and (specific) star formation rate (e.g. Bouché et al. 2006; Chen et al. 2010a; Bordoloi et al. 2011; Lan, Ménard, \& Zhu 2014; Rubin et al. 2018), indicating that massive star-forming galaxies have a relatively dense and/or extended CGM compared to low-mass passive galaxies. The CGM is also related to galaxy orientation (e.g. Steidel et al. 2002; Kacprzak, Churchill, \& Nielsen 2012), originating from that gas accretion on to galaxies is preferentially along their major axis, whereas gas outflows along the minor axis.

The CGM is expected to be affected by galaxy environment as well as galaxy properties. The $\mathrm{H}_{\mathrm{I}}$ observations in the local Universe reveal that gas around galaxies has complex structures when the galaxy interacts with neighbour galaxies or the host group/cluster (e.g. Chynoweth et al. 2008; Chung et al. 2009; Wolfe et al. 2013). The combined analysis of internal velocity dispersion of galaxies and strong lensing predicts that the halo size of cluster galaxies is significantly different from that of field galaxies (e.g. Monna et al. 2015, 2017). However, the results from previous studies with the $\mathrm{Mg}$ II doublet do not seem to agree. For example, Lopez et al. (2008) and Padilla et al. (2009) found that strong Mg II absorbers are abundant in cluster central regions, while the numbers of weak absorbers are compatible between cluster and field regions. The authors suggested that many galaxies are in clusters, but their haloes are truncated by cluster environment effects. On the other hand, Chen et al. (2010b) 
reported that the strengths of $\mathrm{Mg}$ II absorption in group environments are similar to those in isolated environments. Bordoloi et al. (2011) pointed out that the extended $\mathrm{Mg}$ II gas around group galaxies could be explained by a superposition of member galaxies (but see Nielsen et al. 2018), implying that the group environment does not have a significant impact on the CGM of individual galaxies.

To systematically study how the CGM is affected by the cluster environment, we construct a large sample of quasar-cluster pairs using data sets from the Sloan Digital Sky Survey (SDSS; York et al. 2000) and detect $\mathrm{Mg}$ II absorption lines in the quasar spectra. The outline of this paper is as follows. We explain the data used, including cluster, quasar and galaxy samples, in Section 2. We describe the methods to measure the equivalent widths (EWs) of $\mathrm{Mg}_{\text {II }}$ lines and to select $\mathrm{Mg}$ I absorbers in Section 3. We present and discuss our findings in Section 4. We summarize the results in Section 5. Throughout, we assume a flat $\Lambda \mathrm{CDM}$ cosmology with $H_{0}=100 \mathrm{~h} \mathrm{~km} \mathrm{~s} \mathrm{Mpc}^{-1}$, $\Omega_{\Lambda}=0.7$ and $\Omega_{m}=0.3$. We omit the terms of ' $-5 \log h$ ' and ' $h{ }^{-1}$, from absolute magnitude and cluster mass units, respectively. All magnitudes are given in the $\mathrm{AB}$ system and all measurement errors are $1 \sigma$.

\section{DATA}

\subsection{Galaxy cluster sample}

We use the redMaPPer (red-sequence Matched-filter Probabilistic Percolation; Rykoff et al. 2014, 2016) catalogue to search for Mg II absorbers associated with galaxy clusters. The redMaPPer is one of the largest and homogeneous samples of galaxy clusters. This catalogue is constructed with sophisticated algorithms by sampling red-sequence galaxies as potential cluster members based on a minimal spectroscopic training set. The redMaPPer richness parameter $\left(\lambda_{\text {red }}\right)$ is well correlated with other proxies of cluster mass and about 90 per cent of member candidates are spectroscopically confirmed to be in the host cluster even at the low richness (e.g. Rines et al. 2018; Sohn et al. 2018). Therefore, although the redMaPPer clusters are photometrically selected, most of them are expected to be genuine clusters.

In this study, we adopt the version 6.3 of redMaPPer catalogue ${ }^{1}$ containing 26 000 galaxy clusters in the SDSS Data Release 8 (DR8; Aihara et al. 2011). The dynamical mass $\left(M_{200}\right)$ and projected velocity dispersion $\left(\sigma_{\text {red }}\right)$ of redMaPPer clusters are estimated from $\lambda_{\text {red }}$ using the scaling relations in table 4 of Rines et al. (2018):

$\log \left(M_{200} / 10^{14.5} \mathrm{M}_{\odot}\right)=0.580 \log \left(\lambda_{\text {red }} / 100\right)+0.005$,

$\log \left(\sigma_{\text {red }} / 700 \mathrm{~km} \mathrm{~s}^{-1}\right)=0.240 \log \left(\lambda_{\text {red }} / 100\right)+0.046$.

Then, their virial radius $\left(r_{200}\right)$ is converted from $\sigma_{\text {red }}$ with the formula of Carlberg et al. (1997):

$r_{200} / \mathrm{Mpc}=\frac{\sqrt{3} \sigma_{\mathrm{red}}}{10 H\left(z_{\mathrm{red}}\right)}$,

where $z_{\text {red }}$ is the redMaPPer cluster redshift from the photometric redshifts of member candidates and $H\left(z_{\text {red }}\right)$ is the Hubble parameter at $z_{\text {red }}$ (Peebles 1993).

Figure 1 displays the $\lambda_{\text {red }}$ and $z_{\text {red }}$ distributions. We focus on 6179 clusters, surrounded by red dashed lines, at $z_{\text {red }}=0.34-0.53$ and $\lambda_{\text {red }} \geqq 40$, corresponding to $M_{200} \geqq 1.88 \times 10^{14} \mathrm{M}_{\odot}$. The lower limit of $\lambda_{\text {red }}$ is necessary to guarantee the scaling relations of

\footnotetext{
1 http://risa.stanford.edu/redmapper/
}

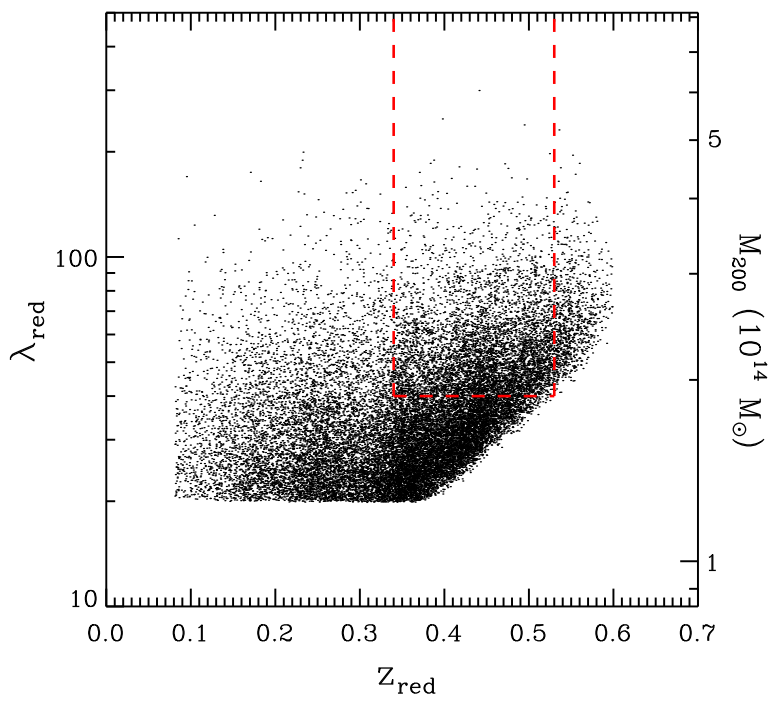

Figure 1. Richness parameter versus redshift diagram of redMaPPer galaxy clusters. Red dashed lines denote our selection criteria.

Rines et al. (2018): their figures 9 and 11 show that the relations are not reliable at $\lambda_{\text {red }}<40$. The upper limit of $z_{\text {red }}$ is for making a volume-limited sample at $\lambda_{\text {red }} \geqq 40$. The lower limit of $z_{\text {red }}$ is determined to ensure that the $\mathrm{Mg}$ II doublet is comfortably within the BOSS (Baryon Oscillation Spectroscopic Survey; Dawson et al. $2013)$ spectral coverage $(3650-10400 \AA)^{2}$ at $z_{\text {red }}-5 \sigma_{\text {red }}\left(1+z_{\text {red }}\right) / c<$ $z<z_{\text {red }}+5 \sigma_{\text {red }}\left(1+z_{\text {red }}\right) / c$, where $c$ is the speed of light, which is the redshift range where we search for absorbers $\left(\Delta v / \sigma_{\text {red }}<5\right.$; the search window hereafter).

\subsection{Quasar sample}

We use the quasar catalogue of Pâris et al. (2018), which is from the SDSS DR14 (Abolfathi et al. 2018) and contains 526 000 quasars. Among these, 15 and 85 per cent were observed with SDSS and BOSS spectrographs, respectively. Their optical spectra are downloadable from the SDSS Science Archive Server ${ }^{3}$.

By cross-matching the quasar and cluster catalogues, we search for quasars with projected distance from the centre of each cluster centre smaller than $5 r_{200}$. This distance is large enough to cover both cluster to field environments so that we can examine any difference between the two environments. We find 96759 quasars (including duplicates) in and around the clusters and hereafter call them quasarcluster pairs for convenience. There is little difference in the redshift distribution of quasars $\left(z_{\mathrm{QSO}}\right)$ between parent and matched samples. In Figure 2, we present the number density and $g$-band point spread function magnitudes of the background quasars as a function of the cluster-centric radius normalized by $r_{200}\left(R / r_{200}\right)$. The mean number density is $48.23 \pm 0.16$ per $\mathrm{deg}^{2}$ and typical range (68 per cent enclosure) of $g$-band magnitudes is 19.54-21.71 mag. Because

2 When using quasar spectra obtained with the SDSS spectrograph (3800$9200 \AA$ ), the redshift cut of $z_{\text {red }} \geqq 0.34$ may not be enough to securely measure the $\mathrm{Mg}$ II doublet. If we remove the SDSS spectrograph data or strictly narrow the redshift range to $z_{\text {red }}=0.39-0.53$, the number of quasar-cluster pairs is reduced by 14 or 26 per cent, respectively. This does not change the main results of this study, but makes our conclusion less significant.

${ }^{3}$ http://dr16.sdss.org/sas/dr16/sdss(eboss)/spectro/redux/ 


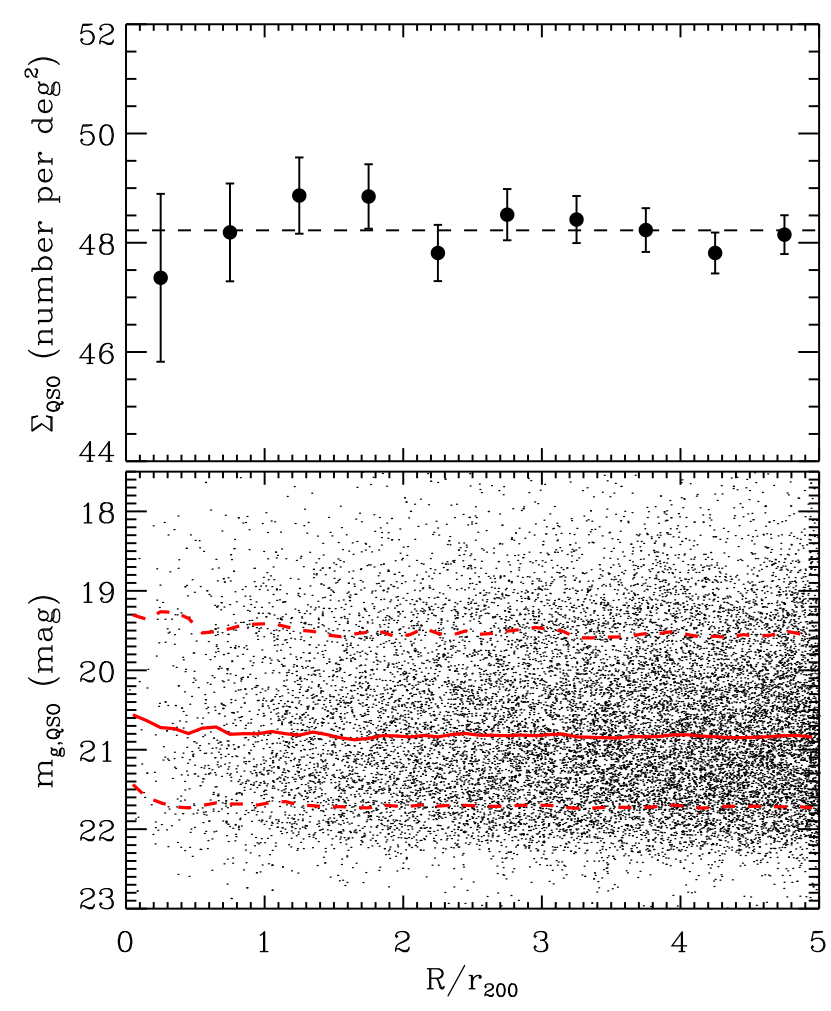

Figure 2. Number density (top) and $g$-band magnitudes (bottom) of quasars cross-matched with redMaPPer clusters as a function of the cluster-centric radius normalized to its virial radius. In the top panel, the horizontal dashed line is the mean number density and error bars are calculated from the binomial statistics. In the bottom panel, the red solid line indicates the sliding median and the dashed lines enclose 68 per cent of quasars. For clarity, (randomly selected) 20 per cent of quasars are presented.

the quasars and clusters are selected independently from each other, the quasar properties show no systematic variation with the clustercentric radius.

\subsection{Galaxy sample}

We also compile galaxy data using a photometric sample of galaxies in the SDSS DR16 (Ahumada et al. 2020). Their redshift information is obtained from the SDSS database and is supplemented from literature (see Hwang et al. 2010, 2014, for details). At $R / r_{200}<$ 5 , the numbers of galaxies in the photometric and spectroscopic samples are about 12.26 million and 486000 , respectively. The spectroscopic completeness as a function of $r$-band Petrosian magnitude $\left(m_{r}\right)$ is shown in the left panel of Figure 3. The limiting magnitude of SDSS main galaxy survey is $m_{r}=17.77$ (Strauss et al. 2002). However, the completeness is not small even at $m_{r}>17.77$ because of other subprograms targeting faint extra-galaxies such as the Luminous Red Galaxy (LRG; Eisenstein et al. 2001), Emission Line Galaxy (ELG; Comparat et al. 2016), SPectroscopic IDentification of ERosita Sources (SPIDERS; Clerc et al. 2016) and Extended Baryon Oscillation Spectroscopic Survey (eBOSS; Dawson et al. 2016). We note that a significant fraction of redMaPPer clusters were not covered by the main galaxy survey, which makes the completeness for the cluster regions reaches only half even at $m_{r}<17.77$.

Among 486000 galaxies with redshift, 64716 galaxies are found to be within the search window. The primary source of them is the LRG sample (79 per cent) rather than the main galaxy sample (10 per cent) because of redshift range. Their $m_{r}$ and $(g-r)$ model colour ranges are about 18.0-20.5 and 1.0-2.0 mag, respectively. When fixing $m_{r}$ from 18.0 to $20.5 \mathrm{mag}$, we can see the colour dependence of spectroscopic completeness as shown in the middle panel of Figure 3: high completeness in the red colour range connected to the selection criteria for LRGs. At $m_{r}<17.77$, dominated by the main galaxy sample, this colour dependence disappears because there is no colour selection for the main galaxy sample. At $m_{r}=18.0-20.5$ and $(g-r)$ $=1.0-2.0$, we can perceive the radial dependence of completeness (the solid line in the right panel) as well. The high completeness in the cluster region may originate mainly from that the redMaPPer cluster finding technique prefers galaxies with redshift as member galaxies, especially for the central galaxy (see section 8 of Rykoff et al. 2014), and partly from that some redMaPPer cluster galaxies are targeted in the SPIDERS programs.

We regard 64716 galaxies within the search window as galaxies associated with an ensemble of redMaPPer clusters (the redMaPPer cluster galaxies hereafter) and plot their colour-magnitude diagram in Figure 4. The absolute magnitude $M_{r}$ and $(g-r)_{0}$ colour are obtained after $K$-correction (Blanton \& Roweis 2007) and evolution correction (Tegmark et al. 2004). Because most of them are LRGs, the so-called red sequence is well noticeable, whereas the blue cloud is not. Using galaxies brighter than $M_{r}=-21$, at which the number of galaxies per mag is peaked, we fit the red sequence to a linear relation (green line):

$(g-r)_{0}=-0.028 M_{r}+0.397(1 \sigma$ scatter $=0.107)$.

We then define the four subsamples of cluster galaxies according to their $M_{r}$ and colour deviation from the sequence $\Delta(g-r)_{0}$, which are

bright-red: $-23 \leqq M_{r}<-22 \&-0.1 \leqq \Delta(g-r)_{0}<+0.1$, faint-red: $-22 \leqq M_{r}<-21 \&-0.1 \leqq \Delta(g-r)_{0}<+0.1$,

bright-blue: $-23 \leqq M_{r}<-22 \&-0.6 \leqq \Delta(g-r)_{0}<-0.2$, and faint-blue: $-22 \leqq M_{r}<-21 \&-0.6 \leqq \Delta(g-r)_{0}<-0.2$. These subsamples include 4919, 24615,395 and 2523 galaxies. The typical stellar masses ${ }^{4}$ of the bright and faint galaxies are $4.4-7.8 \times 10^{11}$ and $1.4-4.0 \times 10^{11} \mathrm{M}_{\odot}$, respectively. The specific star formation rates of the red and blue galaxies are $10^{-12.4}-10^{-11.4}$ and $10^{-10.4}-10^{-9.6} \mathrm{yr}^{-1}$. Their apparent $m_{r}$ and $(g-r)$ ranges are denoted in the right panel of Figure 3. The overall completeness in $R / r_{200}$ bins for the bright-red, faint-red, bright-blue and faint-blue galaxies is also presented with red, orange, purple and cyan circles, respectively. The completeness differs between the subsamples (high completeness in the bright/red galaxies), but has a similar radial trend (higher completeness in the inner region).

\section{ANALYSIS}

To detect $\mathrm{Mg}$ II absorption features in the quasar spectrum, we first estimate the quasar continuum. After masking bad pixels flagged by the SDSS pipeline, we use a simple smoothing method with a median filter. The filter size is changed from 15 to 80 times the instrumental resolution $\left(\sim 1.0 \AA ; \sim 70 \mathrm{~km} \mathrm{~s}^{-1}\right.$ for both SDSS and BOSS spectrographs) according to the $\mathrm{S} / \mathrm{N}$ at each pixel (i.e. 15 times at $\mathrm{S} / \mathrm{N}>100$ and 80 times at $\mathrm{S} / \mathrm{N}<5$ ), resulting in an adaptive smoothing effect. This is determined empirically to be small enough so that the global

4 The stellar mass and star formation rate are drawn from the MPA-JHU value-added galaxy catalogues (Gallazzi et al. 2005; Salim et al. 2007). 

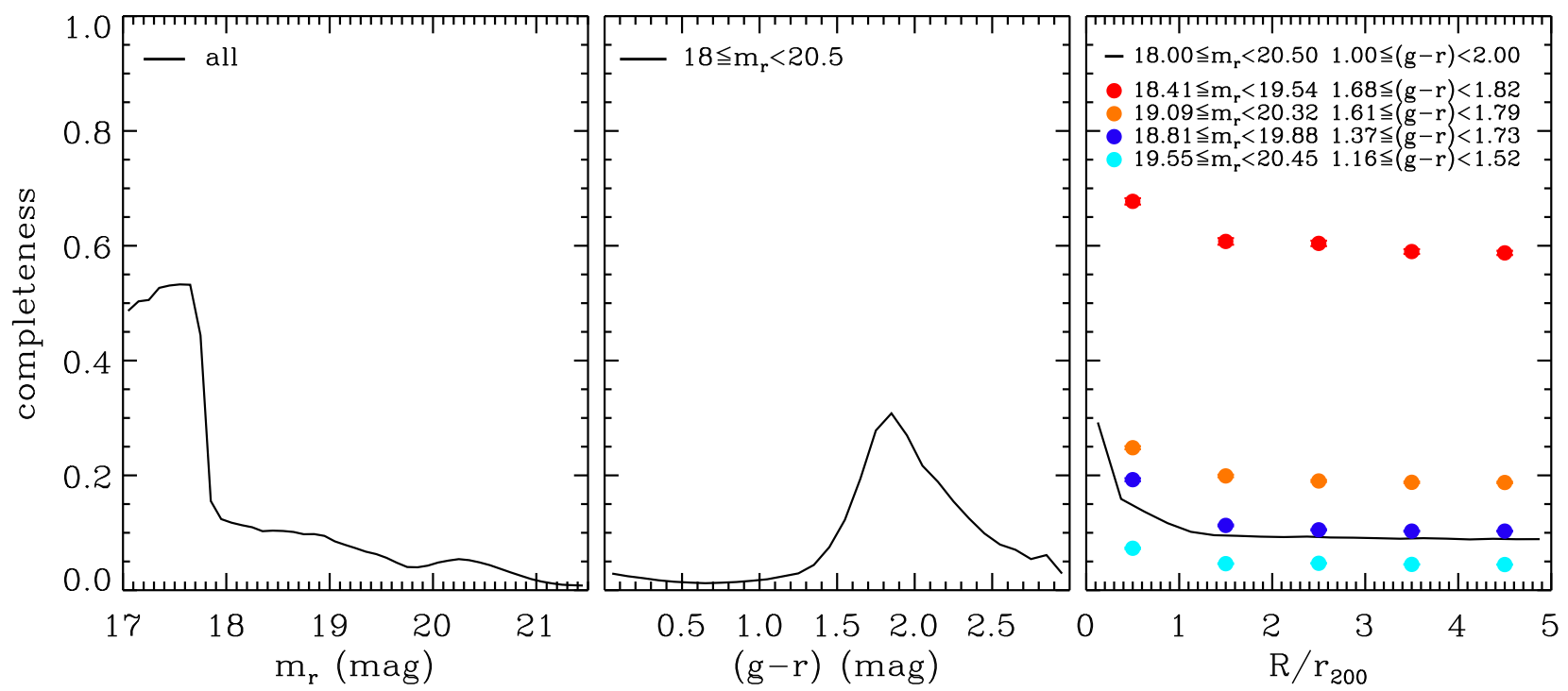

Figure 3. Completeness of the spectroscopic sample of galaxies in and around redMaPPer clusters as functions of $r$-band Petrosian magnitude (left), $(g-r$ ) model colour (middle) and cluster-centric radius (right). In the middle panel, galaxies with $m_{r}=18.0-20.5$ are used to unveil the colour dependence of completeness in galaxies within our search window. In the right panel, the $m_{r}$ and $(g-r)$ cuts are denoted for each case. The solid line is for all galaxies within the search window. The red, orange, purple and cyan circles are for the bright-red, faint-red, bright-blue and faint-blue subsamples, respectively.

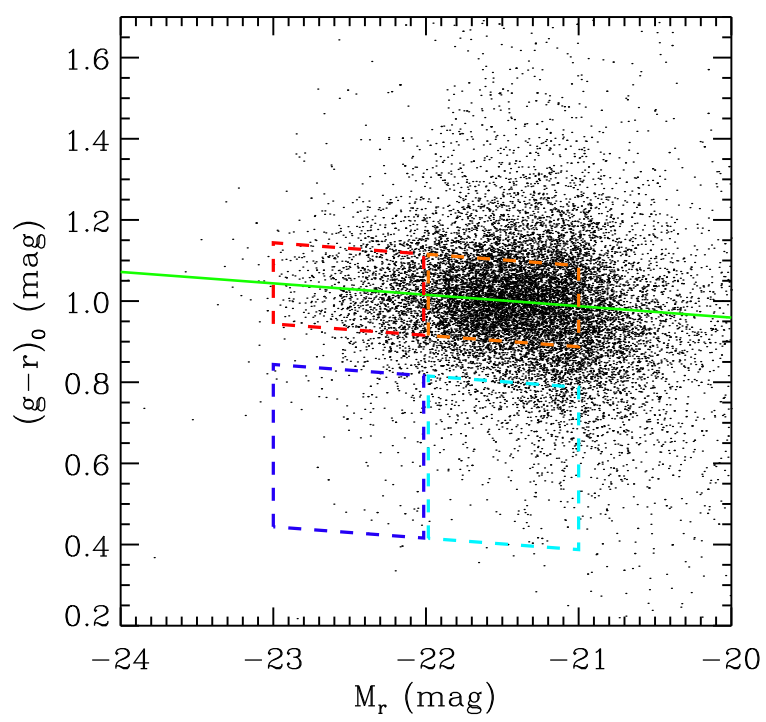

Figure 4. Colour-magnitude diagram of galaxies associated with the redMaPPer clusters. The green line stands for the red sequence. The red, orange, purple and cyan boxes represent the selection criteria of bright-red, faint-red, brightblue and faint-blue galaxies, respectively. For clarity, (randomly selected) 30 per cent of galaxies are presented.

spectral shape and broad emission lines of background quasar should be included in the continuum estimation and to be large enough so that narrow line features such as the $\mathrm{Mg}_{\text {II }}$ doublet from foreground absorbers should not be included. The left panels of Figure 5 show three examples of the quasar spectra along with the continuum fitting result.

Within the search window, we attempt to measure the EWs of
$\mathrm{Mg}_{\text {II }}$ doublet lines $\left(E W_{\lambda 2796} \& E W_{\lambda 2803}\right)$ in every step of $\Delta z=5 \times$ $10^{-5}$ ( $\sim 1 / 7$ of instrumental resolution). The continuum-normalized observed spectrum is transformed to the rest-frame at a potential redshift, and then renormalized by the local continuum (determined from a linear fitting around the doublet at 2765-2785 and 2815$2835 \AA$ ) to remove the remaining residual. Using the MPFIT/IDL package (Markwardt 2009), the Mg II doublet is modelled with two Gaussians, as shown in the right panels of Figure 5. The wavelength separation of the two Gaussians (usually 7.2 $\AA$ for the Mg II doublet) is allowed to vary with a tolerance of \pm 2 times the (rest-frame) resolution. The Gaussians have the same linewidth, which is limited from 90 per cent of the resolution to $3.1 \AA^{5}$. Because the line ratio of the doublet $E W_{\lambda 2796} / E W_{\lambda 2803}$ is theoretically bounded between 2 (unsaturated) and 1 (completely saturated), we keep the measurement with $1-\sigma_{\text {ratio }} \leqq E W_{\lambda 2796} / E W_{\lambda 2803} \leqq 2+\sigma_{\text {ratio }}$, where $\sigma_{\text {ratio }}$ is the ratio error calculated using the uncertainties in Gaussian parameters. The $\mathrm{S} / \mathrm{N} \geqq 3$ is taken as the detection threshold for both $E W_{\lambda 2796}$ and $E W_{\lambda 2803}$. There is another condition that the height of each Gaussian is three times larger than the flux fluctuation (root mean square) around the Mg II doublet, which means that the doublet should be a major line feature at least around it, helpful for preventing false detections (see also next paragraph). More than one $\mathrm{Mg}$ II absorber could be identified in a given spectrum. Therefore, as we measure EWs by increasing the redshift for each step, we keep all the redshifts that give local maxima of the $\mathrm{S} / \mathrm{N}$ for total $\mathrm{EW}$ as the absorber redshifts $\left(z_{\text {abs }}\right)$. This automated procedure results 362 possible $\mathrm{Mg}$ II absorber candidates from 82360 quasar spectra.

\footnotetext{
5 By considering the uncertainty of instrumental resolution information provided by the pipeline, we regard the 90 per cent value as the minimum width of $\mathrm{Mg}$ II lines. When adopting the 100 per cent value as the lower limit, we lose about 20 per cent of absorbers. If the full width at half maximum (FWHM) of $\mathrm{Mg}_{\text {II }}$ lines is larger than the separation, it is difficult to identify the doublet based on our scheme. We thus focus only on narrow (velocity dispersion $\sigma_{\mathrm{Mg} \text { II }}<200 \mathrm{~km} \mathrm{~s}^{-1}$ ) absorption line systems in this study.
} 

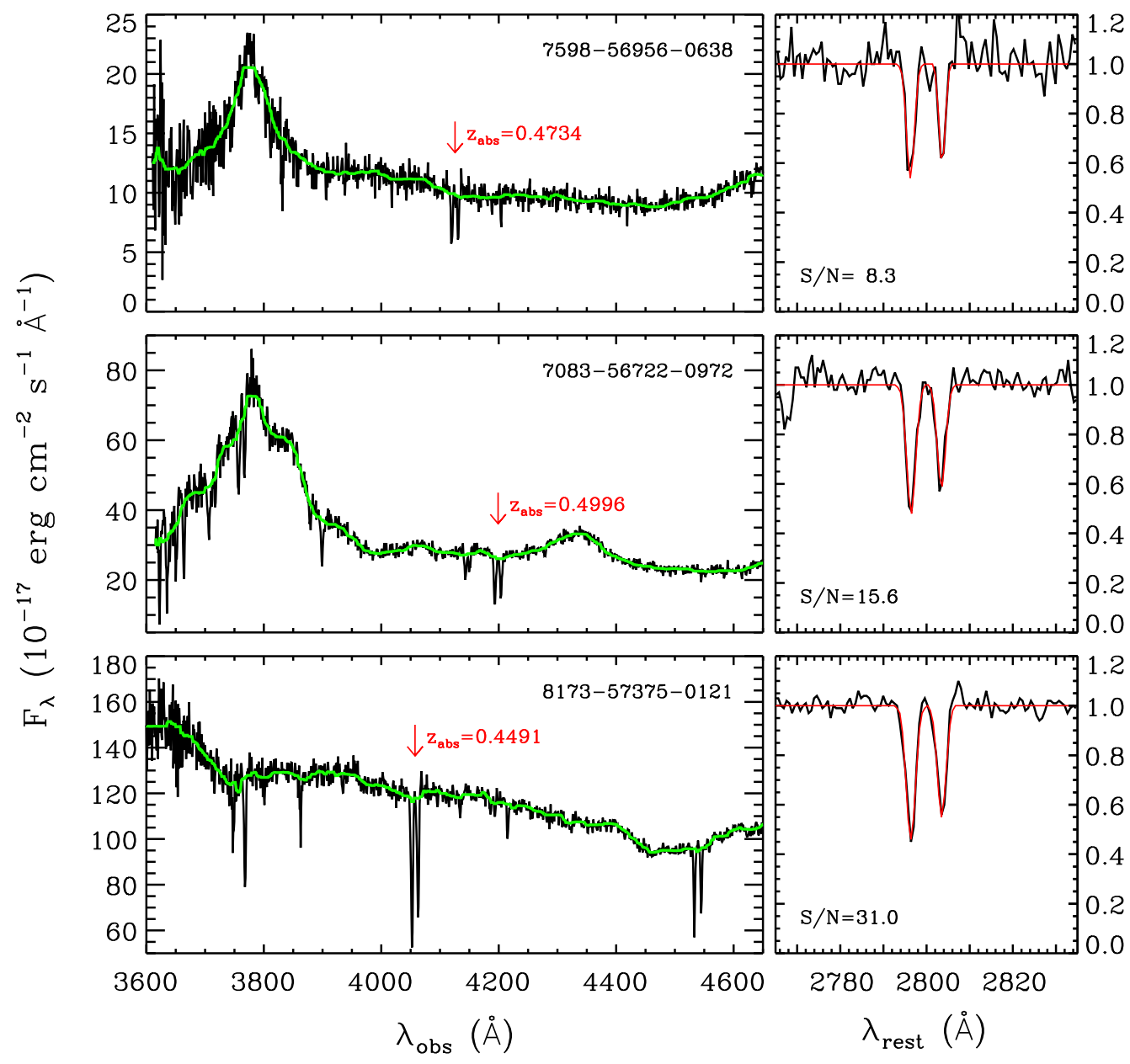

Figure 5. Examples of $\mathrm{Mg}$ II doublet detected spectra. In the left panels, the observed spectrum (ID denoted in the top-right corner) and median-smoothed global continuum are in black and green, respectively. We mark the redshift at which the $\mathrm{Mg}$ II absorber is found with a downward arrow. In the right panels, the (rest-frame) continuum-normalized spectrum and the two-Gaussian model fitting result are in black and red, respectively. The $\mathrm{S} / \mathrm{N}$ of total EW of Mg II lines is presented in the bottom-left corner.

Because a substantial fraction of the 362 possible candidates can be contaminated by false detections, we visually inspect them to select more reliable ones. We exclude the measurements with the Gaussian fits not matched to the actual line profiles ${ }^{6}$. The measurements based on inaccurate continuum estimates ${ }^{7}$ are also eliminated. As a result, 288 probable candidates remain. In Figure 6, we present the histogram of the values obtained by subtracting 7.2 $\AA$ from the wavelength separation between the two Gaussians and dividing by the instrumental resolution ( $\Delta$ separation/resolution). A significant fraction of our candidates are genuine in the sense that the $\Delta$ separation/resolution distribution is concentrated around zero. If we

\footnotetext{
${ }^{6}$ It is difficult to remove them simply by using a $\chi^{2}$ threshold because there could be adjacent line features.

7 Our continuum estimation is not satisfactory when there are broad absorption lines or many adjacent (narrow) absorption lines. It is also incorrect near the peak (not the wing) of broad emission lines. To handle these cases properly, more detailed modelling is necessary.
}

do not adopt the criterion that the Gaussian heights are three times larger than the flux fluctuation, this concentration becomes much weaker. On the other hand, this distribution has secondary peaks at \pm 2 because of the measurements enforced by the boundary condition. We finally select $197 \mathrm{Mg}$ II absorbers with $\operatorname{abs}(\Delta$ separation/resolution) $\leqq 1$ to further reduce contamination. The number of contaminants in the final list is estimated to be 56 by considering the number of probable candidates with $\operatorname{abs}(\Delta$ separation/resolution $)=1-1.75$ (seven absorbers per bin). Therefore, we expect that the reliability of our identification is $(1-56 / 197) \sim 72$ per cent.

We note that all the $197 \mathrm{Mg}$ II absorbers have redshifts smaller than those of quasars (i.e. $z_{\mathrm{abs}}<z_{\mathrm{QSO}}$ ). Among them, there are four absorbers with $z_{\mathrm{QSO}}-z_{\mathrm{abs}}<0.003$, corresponding to the relative velocity of $\Delta v<600 \mathrm{~km} \mathrm{~s}^{-1}$. Although these absorbers are potentially associated with their quasars (i.e. not intervening absorbers), they are kept in the final list for consistency. On the other hand, there are five absorbers matched with two different clusters. We thus have 202 $\mathrm{Mg}$ II absorber-cluster pairs. In a given single spectrum of quasar, 


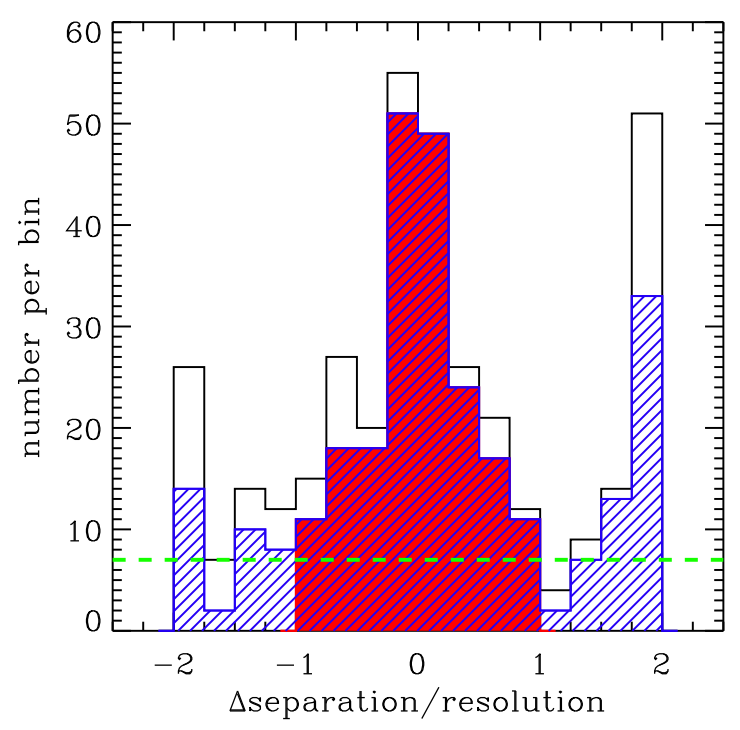

Figure 6. Distribution of the difference between the measured wavelength separation and the intrinsic value of $7.2 \AA$ divided by the instrumental resolution. The black solid, blue hatched and red shaded histograms are for the 362 possible candidates from the automated process, 288 probable candidates through our visual inspection, and $197 \mathrm{Mg}$ II absorbers finally selected, respectively. The green dashed line indicates the estimated contamination level per bin.

there could be multiple absorbers at different redshifts. However, we find no such case in this study due to the low detection rate (only 0.6 per cent even in cluster regions; see Section 4.2).

\section{RESULTS AND DISCUSSION}

\subsection{Absorber properties}

We provide the basic information of $197 \mathrm{Mg}$ II absorbers in Table 1 and plot the measured parameters as functions of $z_{\mathrm{abs}}, R / r_{200}$ and $M_{200}$ in Figure 7 . The sensitivity for detecting $M_{\text {II }}$ absorbers gradually increases with redshift until $z \approx 1.5$ because the SDSS spectroscopic throughput reaches a maximum at $\sim 7000 \AA$. However, we find no correlation between $E W_{\lambda 2796}$ and $z_{\text {abs }}$ in the sense that the probability of obtaining the given correlation by chance in the Spearman test, shown in the top-left panel, is not small enough. This suggests that there is no significant selection bias within our redshift range. We also note that the measured parameters are correlated neither with $R / r_{200}$ nor $M_{200}$; this dependence does not change even if we add the sample of Lopez et al. (2008). There are publicly available catalogues of $\mathrm{Mg}$ II absorbers, which are selected regardless of their environment (e.g. Zhu \& Ménard 2013; Raghunathan et al. 2016). The 36 absorbers are also included in the catalogue of Zhu \& Ménard (2013). For these common absorbers, two measurements are consistent with each other (see Appendix A for more details).

The typical ranges of $E W_{\lambda 2796}, E W_{\lambda 2796} / E W_{\lambda 2803}$ and $\sigma_{\mathrm{Mg} \text { II }} 8$ are $0.97-2.52 \AA, 0.95-1.49$ and $37-105 \mathrm{~km} \mathrm{~s}^{-1}$, respectively. The $\mathrm{Mg}$ II absorbers are often classified into weak and strong systems

\footnotetext{
8 We obtain the intrinsic linewidth by subtracting the 90 per cent of instrumental resolution in quadrature.
}

using the division value of $E W_{\lambda 2796}=0.3 \AA$ (e.g. Lopez et al. 2008). If we follow this classification scheme, all our absorbers are strong systems. The eight absorbers are even ultra-strong $\left(E W_{\lambda 2796}\right.$ $>3 \AA$ ). We note that, based on the completeness test result of Zhu \& Ménard (2013), more than 60 per cent of Mg II absorbers with $E W_{\lambda 2796}>1.0 \AA$ could be detected around $\mathrm{z}=0.45$ from the SDSS quasar spectra, but less than 10 per cent for $E W_{\lambda 2796}<0.3 \AA$. The $E W_{\lambda 2796} / E W_{\lambda 2803}$ distribution is peaked at $\sim 1.2$, indicating that most of the doublets are saturated. About 80 per cent of absorbers have $\sigma_{\mathrm{Mg} \text { II }}<100 \mathrm{~km} \mathrm{~s}^{-1}$, which are compatible with gas velocity dispersion around ELGs rather than LRGs (Lan \& Mo 2018). These absorber properties are generally in agreement with those found in the large samples of Zhu \& Ménard (2013) and Raghunathan et al. (2016).

\subsection{Radial distribution in and around galaxy clusters}

In the left panel of Figure 8, we present the $\mathrm{Mg}$ II detection rate per quasar, the number ratio of $\mathrm{Mg}_{\mathrm{II}}$ absorbers to quasars, in each $R / r_{200}$ bin. Because a small number of quasars are matched with distant clusters that are apparently small, the contribution from distant clusters is underestimated when calculating the detection rate. It is also underestimated in the clusters where the quasar number density is low ${ }^{9}$. Therefore, we set the scaling factor for each cluster so that the adjusted number of quasars matched with a cluster is exactly proportional to the square of the cluster physical size and the total number of quasars remains the same. The raw and corrected detection rates are denoted by open and filled circles, respectively. The reason why the corrected values are slightly high is because a large number of absorbers are detected at high- $z$ due to the spectral characteristics mentioned above. We also need to consider that the absorbers are easily detected when using bright quasars. However, the negligible dependence of quasar magnitudes on $R / r_{200}$, as shown in Figure 2, enables us to assume that the radial trend of the detection rate reflects well the actual distribution of $\mathrm{Mg}$ II absorbers. In this plot, we find that the detection rate increases as the cluster-centric radius decreases: on average $0.59 \pm 0.13$ per cent at $R / r_{200}<1$ and $0.22 \pm 0.03$ per cent at $R / r_{200} \geqq 4$, indicating that $\mathrm{Mg}_{\text {II }}$ absorbers are $2.70 \pm 0.66$ times more abundant in the clusters.

We show the normalized number density of cluster galaxies $\Sigma_{\text {gal }}$ in the right panel. The bright-red, faint-red, bright-blue and faintblue galaxies are denoted by the red, orange, blue and cyan squares, respectively. The raw and spectroscopic completeness corrected values are presented in the open and filled symbols. The radial variations of $\Sigma_{\text {gal }}$ depend on galaxy magnitude and colour: $17.17 \pm 0.83$, $7.14 \pm 0.41,5.41 \pm 1.08$ and $3.88 \pm 0.34$ times higher at $R / r_{200}<1$ than at $R / r_{200} \geqq 4$ for the bright-red, faint-red, bright-blue and faint-blue galaxies, following the well-known relation that a large fraction of massive passive galaxies are in clusters (e.g. Park \& Hwang 2009). Meanwhile, these values are larger than $2.70 \pm 0.66$ found in the $\mathrm{Mg}$ II absorbers. If we assume that the majority of absorbers are connected to the CGM of cluster galaxies in particular for the bright-blue galaxies, the absorber-to-galaxy density ratio at $R / r_{200}<1$ is $0.50 \pm 0.16$ times that at $R / r_{200} \geqq 4$. Because this ratio is proportional to the projected surface area of $\mathrm{Mg}_{\text {II }}$ halo (i.e. ratio $\propto$ halo $\operatorname{size}^{2}$ ), the value less than one means that a typical halo size of cluster galaxies is smaller than that of field galaxies. In Figure 9, we present the halo

9 The SDSS quasar survey is not uniform across the sky. The number density 

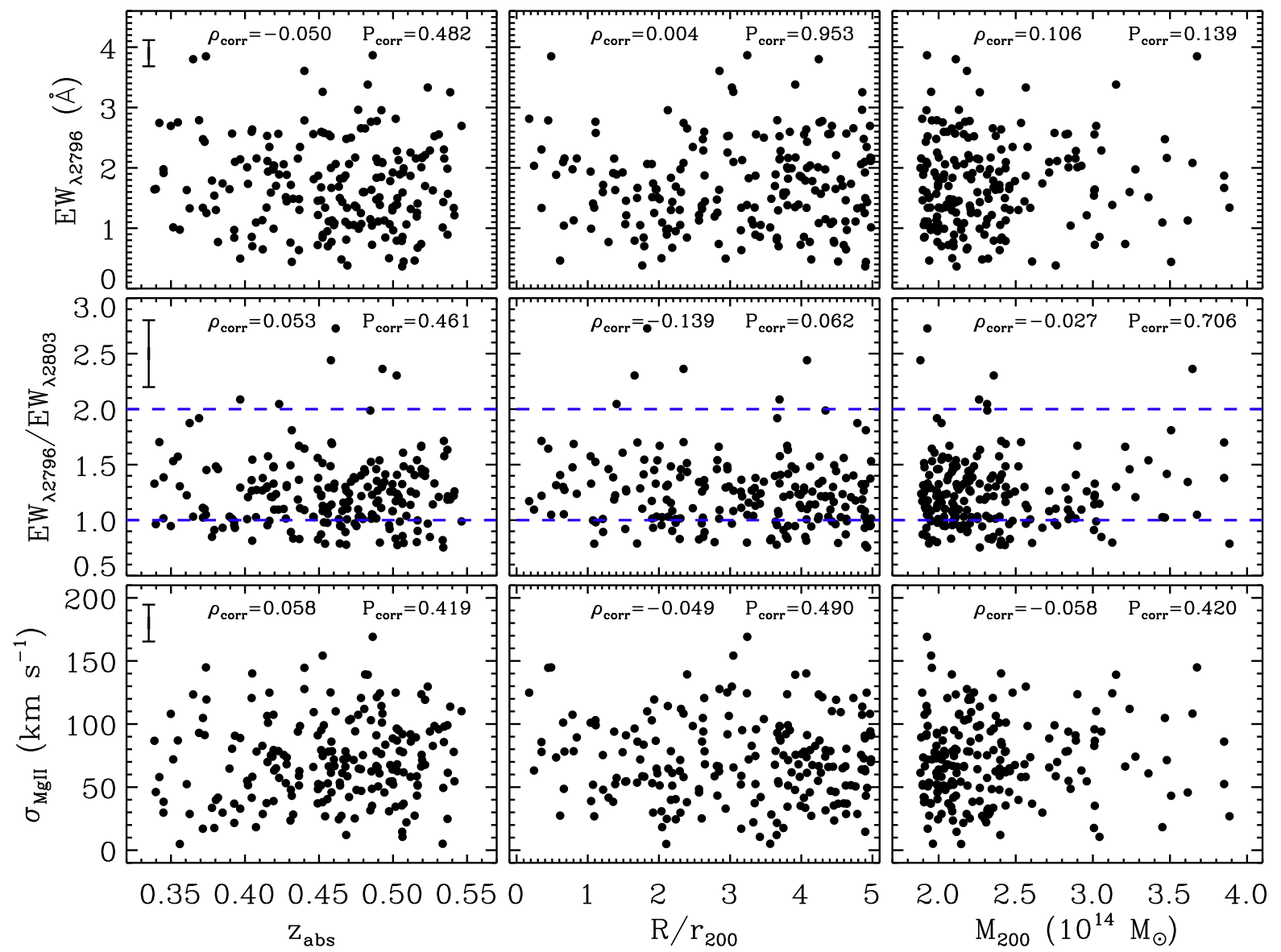

Figure 7. $\mathrm{Mg}$ II absorber properties as functions of absorber redshift (left), cluster-centric radius (middle) and cluster mass (right). The EW of Mg II $\lambda 2796$, doublet line ratio and velocity dispersion of $\mathrm{Mg}$ II lines are presented in the top, middle and bottom panels, respectively. Their median errors are denoted in the top-left corner. The numbers in each panel indicate the Spearman rank correlation coefficient $\rho_{\text {corr }}$ and the probability of obtaining the correlation by chance $\mathrm{P}_{\text {corr }}$. The blue horizontal dashed lines are the theoretical limits of the line ratio.
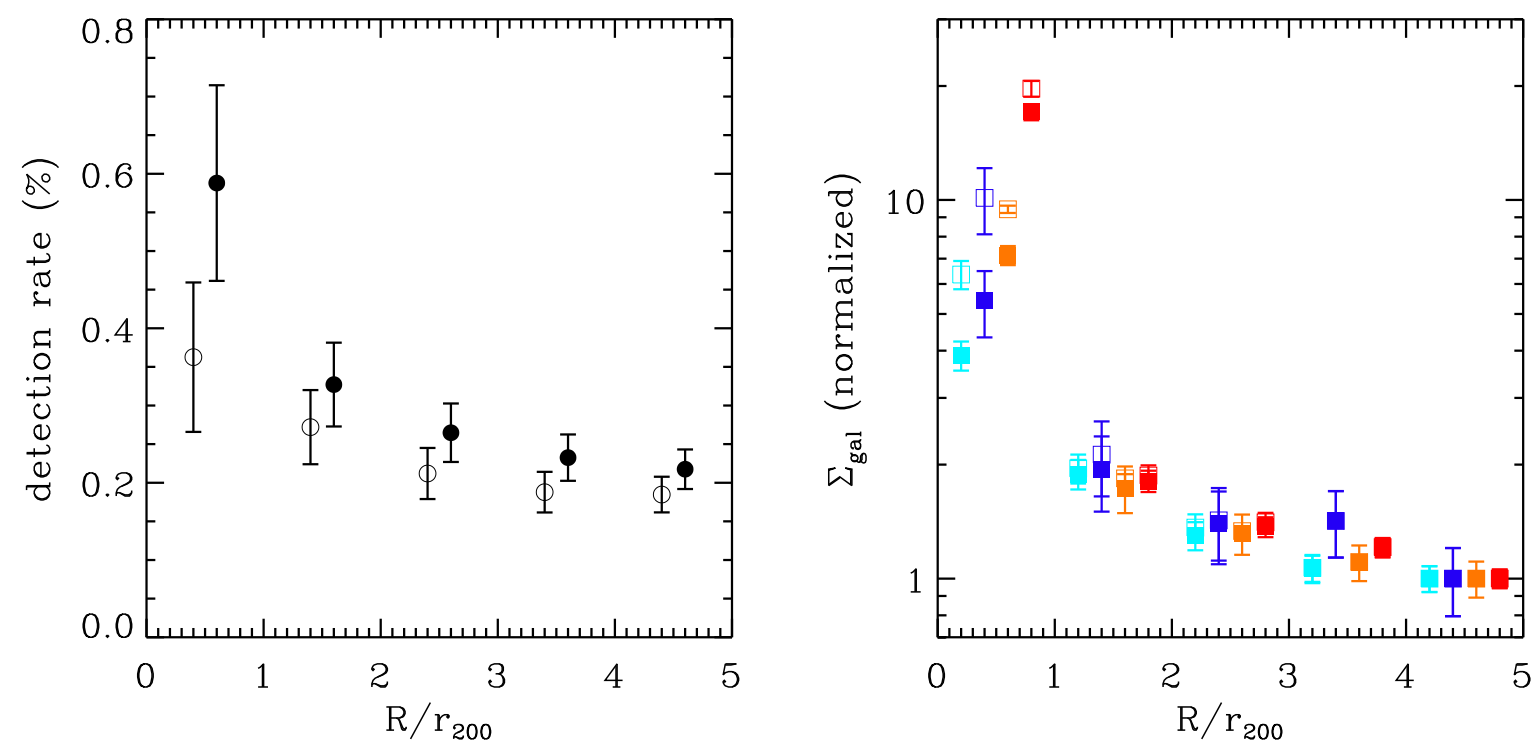

Figure 8. $\mathrm{Mg}$ II absorber detection rate per quasar (left) and the number density of four subsamples of cluster galaxies, normalized by the value at $R / r_{200}>4$ (right), as a function of cluster-centric radius. In the right panel, the red, orange, blue and cyan squares are for the bright-red, faint-red, bright-blue and faint-blue galaxies, respectively. The open and filled circles/squares indicate the raw and corrected values. These symbols are properly separated for better visibility. 
Table 1. Basic information of $197 \mathrm{Mg}$ II absorbers. Columns 1-4 are from the quasar catalogue of Pâris et al. (2018). Columns 5-8 are our measurements and errors. The absorber redshift errors are typically 0.0003 . The full table is available online.

\begin{tabular}{cccccccc}
\hline spectrum ID & $\begin{array}{c}\text { RA } \\
\text { deg. }\end{array}$ & $\begin{array}{c}\text { Dec } \\
\text { deg. }\end{array}$ & $z_{\text {QSO }}$ & $z_{\text {abs }}$ & $\begin{array}{c}E W_{\lambda 2796} \\
\AA\end{array}$ & $E W_{\lambda 2796} / E W_{\lambda 2803}$ & $\begin{array}{c}\sigma_{\mathrm{Mg}_{\text {II }}} \\
\mathrm{km} \mathrm{s}^{-1}\end{array}$ \\
\hline $7830-57043-0126$ & 034.956963 & 01.454112 & 1.4497 & 0.3736 & $3.85 \pm 0.63$ & $1.05 \pm 0.27$ & $144.9 \pm 26.8$ \\
$6468-56311-0076$ & 151.811924 & 31.772667 & 3.3370 & 0.3716 & $2.48 \pm 0.31$ & $1.02 \pm 0.17$ & $104.8 \pm 16.5$ \\
$4608-55973-0567$ & 133.845293 & 37.907218 & 2.3020 & 0.4074 & $1.09 \pm 0.14$ & $1.03 \pm 0.18$ & $018.3 \pm 11.2$ \\
$4572-55622-0204$ & 146.842194 & 38.014749 & 2.4010 & 0.4584 & $1.67 \pm 0.22$ & $1.70 \pm 0.39$ & $086.1 \pm 14.6$ \\
$4572-55622-0156$ & 146.997598 & 38.379074 & 2.2780 & 0.4494 & $1.87 \pm 0.25$ & $1.38 \pm 0.30$ & $052.4 \pm 11.9$ \\
\hline
\end{tabular}

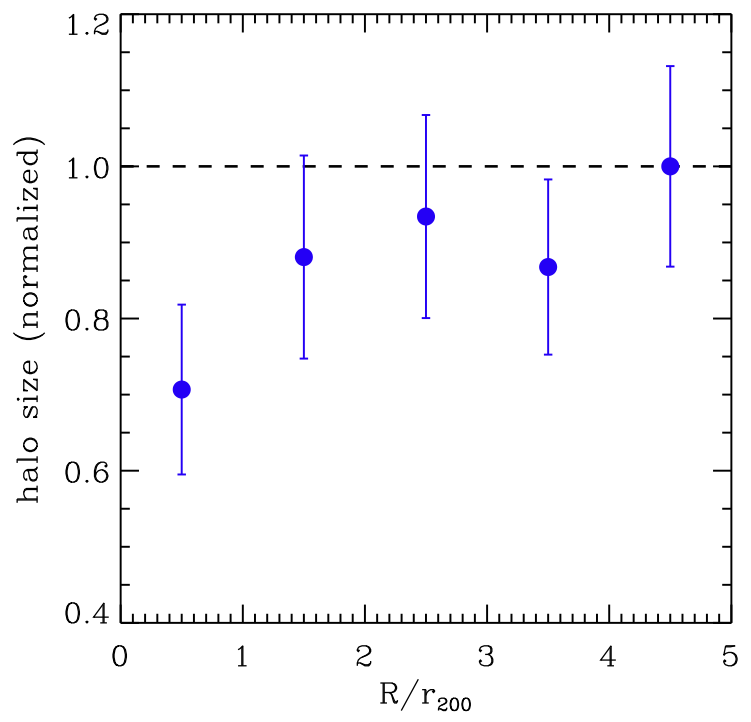

Figure 9. Relative $\mathrm{Mg}_{\text {II }}$ halo size variation on cluster-centric radius. The horizontal dashed line is overplotted to guide the eye. This result is based on the assumption that $\mathrm{Mg}$ II absorbers are mostly associated with the CGM of bright-blue galaxies.

size normalized by that at $R / r_{200} \geqq 4$ (the relative halo size hereafter) as a function of $R / r_{200}$ based on the bright-blue galaxies. The relative halo size is $0.71 \pm 0.11$ at $R / r_{200}<1$.

This result is not as dramatic as the argument of Padilla et al. (2009) that $\mathrm{Mg}$ II halo radii can be changed from 50 to $10 \mathrm{kpc}$ by cluster environmental effects. However, they might have overestimated the cluster effect in the sense that they did not take into account the dependency of $\mathrm{Mg}$ II absorption on galaxy colour. The relative halo sizes in the clusters become $0.40 \pm 0.05,0.62 \pm 0.08$ and $0.83 \pm 0.11$ when using the bright-red, faint-red, and faint-blue galaxies, respectively. There is a possibility that we underestimate the cluster effect because weak absorbers (sensitive to environmental effects) are not considered in this study, while Padilla et al. (2009) utilized some weak absorbers from their own observations.

The truncated $\mathrm{Mg}$ II halo in cluster environment is supported by the low covering fraction of Ly $\alpha$ absorption in the CGM of cluster galaxies compared to field galaxies (e.g. Yoon \& Putman 2013; Burchett et al. 2018). To explain this phenomenon, Padilla et al. (2009) suggested the cold gas stripping scenario by the hot intracluster gas. Because we cannot test this scenario with the current data sets, it is difficult to discuss the detailed mechanism of gas stripping: for example, is it driven by ram pressure (Gunn \& Gott 1972) or thermal evaporation (Cowie \& Songaila 1977)? It is also difficult to rule out other types of processes such as strangulation (Larson, Tinsley, \& Caldwell 1980), harassment (Moore et al. 1996), tidal interaction with the cluster potential (Merritt 1984) and high-speed multiple encounters with early-type galaxies (Park \& Hwang 2009).

There are Ly $\alpha$ and C IV absorbers associated with the IGM component of clusters rather than the CGM of cluster galaxies (e.g. Yoon \& Putman 2017; Manuwal et al. 2019). However, Mg II absorbers can hardly trace the IGM because the H I column density of IGM is too low, which is less than $10^{16} \mathrm{~cm}^{-2}$ in most areas (see the hydrodynamic simulation result of Butsky et al. 2019). The IGM contribution is expected to be small, thus not changing our conclusion.

\subsection{Phase-space diagram}

Figure 10 displays the distribution of $\mathrm{Mg}$ II absorbers and redMaPPer cluster galaxies in projected phase space. The $\mathrm{x}$-axis is the projected distance from the cluster centre normalized by the cluster radius $R / r_{200}$, while the y-axis is the line-of-sight velocity difference between the absorber/galaxy and the cluster normalized by the cluster velocity dispersion $\Delta v / \sigma_{\text {red. }}$. The radial variation of spectroscopic completeness for the cluster galaxies is not considered in the phasespace diagram. For a quantitative comparison, we also present the $\Delta v / \sigma_{\text {red }}$ histograms of the two populations at $R / r_{200}<1$ and $\geqq 4$. The $\Delta v / \sigma_{\text {red }}$ distribution is less affected by galaxy magnitude and colours, so we include all the cluster galaxies in the histograms. Both histograms are nearly flat and identical ( $p$-value of the KolmogorovSmirnov test $=0.213$ ) in the field environment (i.e. $R / r_{200} \geqq 4$ ). Inside the clusters, the cluster galaxies are naturally biased towards small $\Delta v / \sigma_{\text {red }}$, even though the number of galaxies with $\Delta v / \sigma_{\text {red }}<1$ is only 1.66 times larger than that with $\Delta v / \sigma_{\text {red }} \geqq 4$ : this may be due to non-negligible uncertainty of $z_{\text {red }}$ (Rines et al. 2018; Sohn et al. 2018). Interestingly, the $\mathrm{Mg}$ II absorbers have rather large $\Delta v / \sigma_{\text {red }}$. As a result, the two histograms are significantly different from each other ( $p$-value $=0.010 ; 2.6 \sigma$ level $)$ in the clusters. It emphasizes that $\mathrm{Mg}$ II absorbers disfavour cluster environments.

If we recalculate the values obtained in Section 4.2 using $\mathrm{Mg}$ II absorbers and cluster galaxies with $\Delta v / \sigma_{\text {red }}<1$, the radial density variations of absorbers and bright-blue galaxies become $1.33 \pm 1.18$ and $5.88 \pm 2.64$, respectively, leading to the change of relative halo size in the clusters from $0.71 \pm 0.11$ to $0.48 \pm 0.24$. This result shows that cluster environmental effects can be evaluated more accurately within the narrower search window. However, it is quite uncertain because there is only one absorber at $R / r_{200}<1$ and with $\Delta v / \sigma_{\text {red }}$ $<1$. 


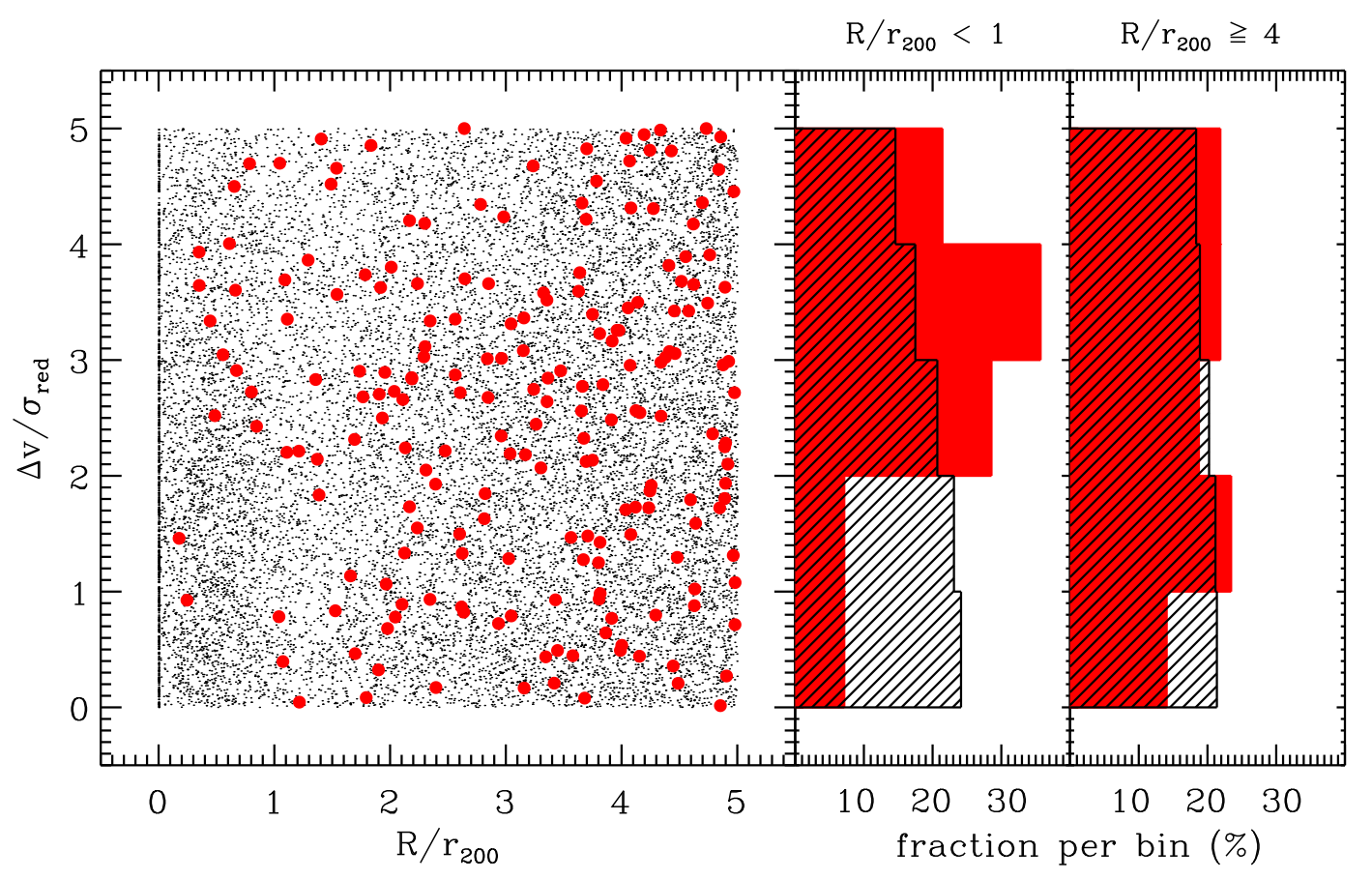

Figure 10. Projected phase-space diagram of $\mathrm{Mg}$ II absorbers and redMaPPer cluster galaxies (left) and their $\Delta v / \sigma_{\text {red }}$ histograms at $R / r_{200}<1$ (middle) and at $R / r_{200}>4$ (right). The black dots and hatched histogram represent the cluster galaxies, while the red circles and shaded histogram are for the Mg II absorbers. In the left panel, (randomly selected) 30 per cent of galaxies are presented for clarity.

\section{SUMMARY}

Using the redMaPPer galaxy clusters and SDSS DR14 quasars, we obtain 96759 quasar-cluster pairs. We then detect intervening Mg II absorption line features in the background quasar spectra. Our main results are as follows.

(i) We find $197 \mathrm{Mg}$ II absorbers with $E W_{\lambda 2796}>0.3 \AA$. The reliability of our identification is estimated to be $\sim 72$ per cent. Their line parameters are not correlated with the cluster properties.

(ii) The $\mathrm{Mg}$ II absorber detection rate per quasar is $2.70 \pm 0.66$ times higher inside the clusters than outside the clusters. It shows that $\mathrm{Mg}$ II absorbers are abundant in clusters compared to in fields.

(iii) Because the galaxy number density is much higher inside the clusters than outside the clusters, the absorber-to-galaxy ratio is relatively low inside the clusters. We suggest that a typical halo size of (bright-blue) galaxies in clusters is $0.71 \pm 0.11$ times that in fields.

It is not easy to study environmental effects on the CGM in detail with individual $\mathrm{Mg}$ II absorbers due to small number statistics. Using stacked spectra of quasars around cluster galaxies, we plan to further investigate how the radial profile of $\mathrm{Mg}$ II absorption strength depends on galaxy and cluster properties.

\section{ACKNOWLEDGEMENTS}

We thank Daeseong Park for discussions in the continuum estimation. We are grateful to an anonymous referee whose comments helped to improve the original manuscript.

Funding for the Sloan Digital Sky Survey IV has been provided by the Alfred P. Sloan Foundation, the U.S. Department of Energy Office of Science and the Participating Institutions. SDSS-IV acknowledges support and resources from the Center for High-Performance Computing at the University of Utah. The SDSS web site is www.sdss.org.

SDSS-IV is managed by the Astrophysical Research Consortium for the Participating Institutions of the SDSS Collaboration including the Brazilian Participation Group, the Carnegie Institution for Science, Carnegie Mellon University, the Chilean Participation Group, the French Participation Group, Harvard-Smithsonian Center for Astrophysics, Instituto de Astrofísica de Canarias, The Johns Hopkins University, Kavli Institute for the Physics and Mathematics of the Universe (IPMU) / University of Tokyo, Lawrence Berkeley National Laboratory, Leibniz Institut für Astrophysik Potsdam (AIP), MaxPlanck-Institut für Astronomie (MPIA Heidelberg), Max-PlanckInstitut für Astrophysik (MPA Garching), Max-Planck-Institut für Extraterrestrische Physik (MPE), National Astronomical Observatories of China, New Mexico State University, New York University, University of Notre Dame, Observatário Nacional / MCTI, The Ohio State University, Pennsylvania State University, Shanghai Astronomical Observatory, United Kingdom Participation Group, Universidad Nacional Autónoma de México, University of Arizona, University of Colorado Boulder, University of Oxford, University of Portsmouth, University of Utah, University of Virginia, University of Washington, University of Wisconsin, Vanderbilt University and Yale University.

\section{DATA AVAILABILITY}

The data underlying this article are available in the article and in its online supplementary material. 


\section{REFERENCES}

Abolfathi B. et al., 2018, ApJS, 235, 42

Adelberger K. L., Shapley A. E., Steidel C. C., Pettini M., Erb D. K., Reddy N. A., 2005, ApJ, 629, 636

Ahumada R. et al., 2020, ApJS, 249, 3

Aihara H. et al., 2011, ApJS, 193, 29

Bergeron J., 1986, A\&A, 155, L8

Blanton M. R., Roweis S., 2007, AJ, 133, 734

Bordoloi R. et al., 2011, ApJ, 743, 10

Bouché N., Murphy M. T., Péroux C., Csabai I., Wild V., 2006, MNRAS, 371,495

Burchett J. N., Tripp T. M., Wang Q. D., Willmer C. N. A., Bowen D. V., Jenkins E. B., 2018, MNRAS, 475, 2067

Butsky I. S., Burchett J. N., Nagai D., Tremmel M., Quinn T. R., Werk J. K., 2019, MNRAS, 490, 4292

Cantalupo S., Arrigoni-Battaia F., Prochaska J. X., Hennawi J. F., Madau P., 2014, Nature, 506, 63

Carlberg R. G., Yee H. K. C., Ellingson E., 1997, ApJ, 478, 462

Charlton J. C., Ding J., Zonak S. G., Churchill C. W., Bond N. A., Rigby J. R., 2003, ApJ, 589, 111

Chen H.-W., Wild V., Tinker J. L., Gauthier J.-R., Helsby J. E., Shectman S. A., Thompson I. B., 2010, ApJ, 724, L176

Chen H.-W., Helsby J. E., Gauthier J.-R., Shectman S. A., Thompson I. B., Tinker J. L., 2010, ApJ, 714, 1521

Chynoweth K. M., Langston G. I., Yun M. S., Lockman F. J., Rubin K. H. R., Scoles S. A., 2008, AJ, 135, 1983

Chung A., van Gorkom J. H., Kenney J. D. P., Crowl H., Vollmer B., 2009, AJ, 138, 1741

Clerc N. et al., 2016, MNRAS, 463, 4490

Comparat J. et al., 2016, A\&A, 592, A121

Cowie L. L., Songaila A., 1977, Nature, 266, 501

Daddi E. et al., 2020, preprint (arXiv:2006.11089)

Dawson K. S. et al., 2013, AJ, 145, 10

Dawson K. S. et al., 2016, AJ, 151, 44

Eisenstein D. et al., 2001, AJ, 122, 2267

Gallazzi A., Charlot S., Brinchmann J., White S. D. M., Tremonti C. A., 2005, MNRAS, 362, 41

Gunn J. E., Gott J. R., 1972, ApJ, 176, 1

Hwang H. S. et al., 2010, A\&A, 522, A33

Hwang H. S., Geller M. J., Diaferio A., Rines K. J., Zahid H. J., 2014, ApJ, 797, 106

Kacprzak G. G., Churchill C. W., Nielsen N. M., 2012, ApJ, 760, L7

Lan T.-W., Ménard B., Zhu G., 2014, ApJ, 795, 31

Lan T.-W., Mo H., 2018, ApJ, 866, 36

Larson R. B., Tinsley B. M., Caldwell C. N., 1980, ApJ, 237, 692

Lehner N., Wotta C. B., Howk J. C., O'Meara J. M., Oppenheimer B. D., Cooksey K. L., 2018, ApJ, 866, 33

Lopez S. et al., 2008, ApJ, 679, 1144

Markwardt C. B., 2009, in Bohlender D. A., Durand D., Dowler P., eds, ASP Conf. Ser. Vol. 411, Astronomical Data Analysis Software and Systems XVIII, Astron. Soc. Pac., San Francisco, p. 251

Manuwal A., Narayanan A., Muzahid S., Charlton J. C., Khaire V., Chand H., 2019, MNRAS, 485, 30

Merritt D., 1984, ApJ, 276, 26

Monna A. et al., 2015, MNRAS, 447, 1224

Monna A. et al., 2017, MNRAS, 465, 4589

Moore B., Katz N., Lake G., Dressler A., Oemler A., 1996, Nature, 379, 613

Nielsen N. M., Kacprzak G. G., Pointon S. K., Churchill C. W., Murphy M. T., 2018, ApJ, 869, 153

Padilla N., Lacerna I., Lopez S., Barrientos L. F., Lira P., Andrews H., Tejos N., 2009, MNRAS, 395, 1135

Pâris I. et al., 2018, A\&A, 613, 51

Park C., Hwang H. S., 2009, ApJ, 699, 1595

Peebles P. J. E., 1993, Princeton Series in Physics (Princeton, NJ: Princeton Univ. Press)

Prochaska J. X. et al., 2013, ApJ, 776, 136

Raghunathan S., Clowes R. G., Campusano L. E., Söchting I. K., Graham
M. J., Williger G. M., 2016, MNRAS, 463, 2640

Rao S. M., Turnshek D. A., Nestor D. B., 2006, ApJ, 636, 610

Rines K. J., Geller M. J., Diaferio A., Hwang H. S., Sohn J., 2018, ApJ, 862, 172

Rubin K. H. R., Diamond-Stanic A. M., Coil A. L., Crighton N. H. M., Moustakas J., 2018, ApJ, 853, 95

Rykoff E. S. et al., 2014, ApJ, 785, 104

Rykoff E. S. et al., 2016, ApJS, 224, 1

Salim S. et al., 2007, ApJS, 173, 267

Sohn J., Geller M. J., Rines K. J., Hwang H. S., Utsumi Y., Diaferio A., 2018, ApJ, 856, 172

Steidel C. C., Kollmeier J. A., Shapley A. E., Churchill C. W., Dickinson M., Pettini M., 2002, ApJ, 570, 526

Steidel C. C. et al., 2010, ApJ, 717, 289

Strauss M. A. et al., 2002, AJ, 124, 1810

Tegmark M. et al., 2004, ApJ, 606, 702

Tumlinson J., Peeples M. S., Werk J. K, 2017, ARA\&A, 55, 389

Wolfe S. A., Pisano D. J., Lockman F. J., McGaugh S. S., Shaya E. J., 2013, Nature, 497, 224

Yoon J. H., Putman M. E., 2013, ApJ, 772, L29

Yoon J. H., Putman M. E., 2017, ApJ, 839, 117

York D. G. et al., 2000, AJ, 120, 1579

Zhang H., Zaritsky D., Zhu G., Ménard B., Hogg D. W., 2016, ApJ, 833, 276

Zhao Y., Ge J., Yuan X., Zhao T., Wang C., Li X., 2019, MNRAS, 487, 801

Zhu G., Ménard B., 2013, ApJ, 770, 130

\section{APPENDIX A: COMPARISON WITH THE JHU CATALOGUE}

Zhu \& Ménard (2013) provide a large sample of intervening Mg II absorbers. Their data sets ${ }^{10}$ are updated using the SDSS DR7 and DR12, the JHU catalogue hereafter. In the JHU catalogue, we find 124 absorbers associated with the redMaPPer clusters (i.e. $R / r_{200}<$ 5 and $\Delta v / \sigma_{\text {red }}<5$ ). Among them, 67 absorbers satisfy the criteria of $\mathrm{S} / \mathrm{N} \geqq 3$ for both $E W_{\lambda 2796}$ and $E W_{\lambda 2803}$ in their calculation. The 36 absorbers are also found in this study. For these common absorbers, our measurements are fully consistent with those in the JHU catalogue, as shown in Figure A1. The measurement errors are comparable with each other as well. There are 31 absorbers not selected in this study. These do not go through the automated pipeline mentioned in Section 3 mostly because the line strength is weak: the S/N of $E W_{\lambda 2796}$ or $E W_{\lambda 2803}$ is less than 3 in our calculation. The example spectra of the absorbers missed in this study are presented in the top panels of Figure A2. Although we choose the five cases with the highest $\mathrm{S} / \mathrm{N}$ of total $\mathrm{EW}$, the spectra are quite noisy.

On the other hand, among the 197 absorbers in this study, 80 absorbers are not included in the JHU catalogue due to the small sample of quasars used. The 76 absorbers are outside the search window of Zhu \& Ménard (2013). They searched for Mg II absorbers within a restrictive redshift range ${ }^{11}$ to avoid false detections from intervening $\mathrm{C}_{\text {IV }}$ and Milky Way Ca II absorption lines. Among the remaining 41 absorbers, 36 absorbers are found in the JHU catalogue, but five ones are missed. The spectra of the absorbers missed in the JHU catalogue are shown in the bottom panels of Figure A2. We check that these absorbers are not contaminated by Fe II lines (see section 2.2.3 of Zhu \& Ménard 2013). It is beyond the scope of this

10 https://www.guangtunbenzhu.com/jhu-sdss-metal-absorber-catalog

11 Among the 197 absorbers in this study, only 64 absorbers are inside the search window of Zhu \& Ménard (2013). Their method is effective to minimize contamination by considering that $\Delta$ separation/resolution distribution of the 64 absorbers is strongly concentrated at zero. However, it seems to eliminate a significant fraction of real absorbers. 

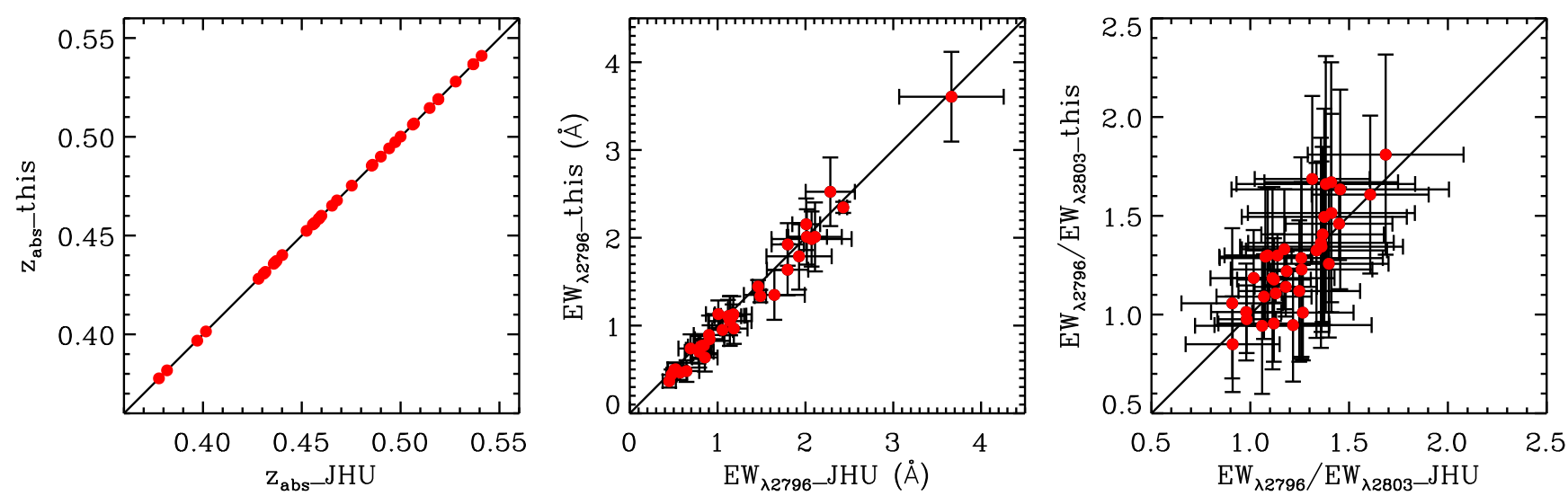

Figure A1. Comparison of the measurements for $\mathrm{Mg}_{\text {II }}$ absorbers between this study and the JHU catalogue. The left, middle and right panels are for the redshift, $\mathrm{EW}$ of $\mathrm{Mg}$ II $\lambda 2796$ and doublet line ratio, respectively. The one-to-one relation (solid line) is overplotted.
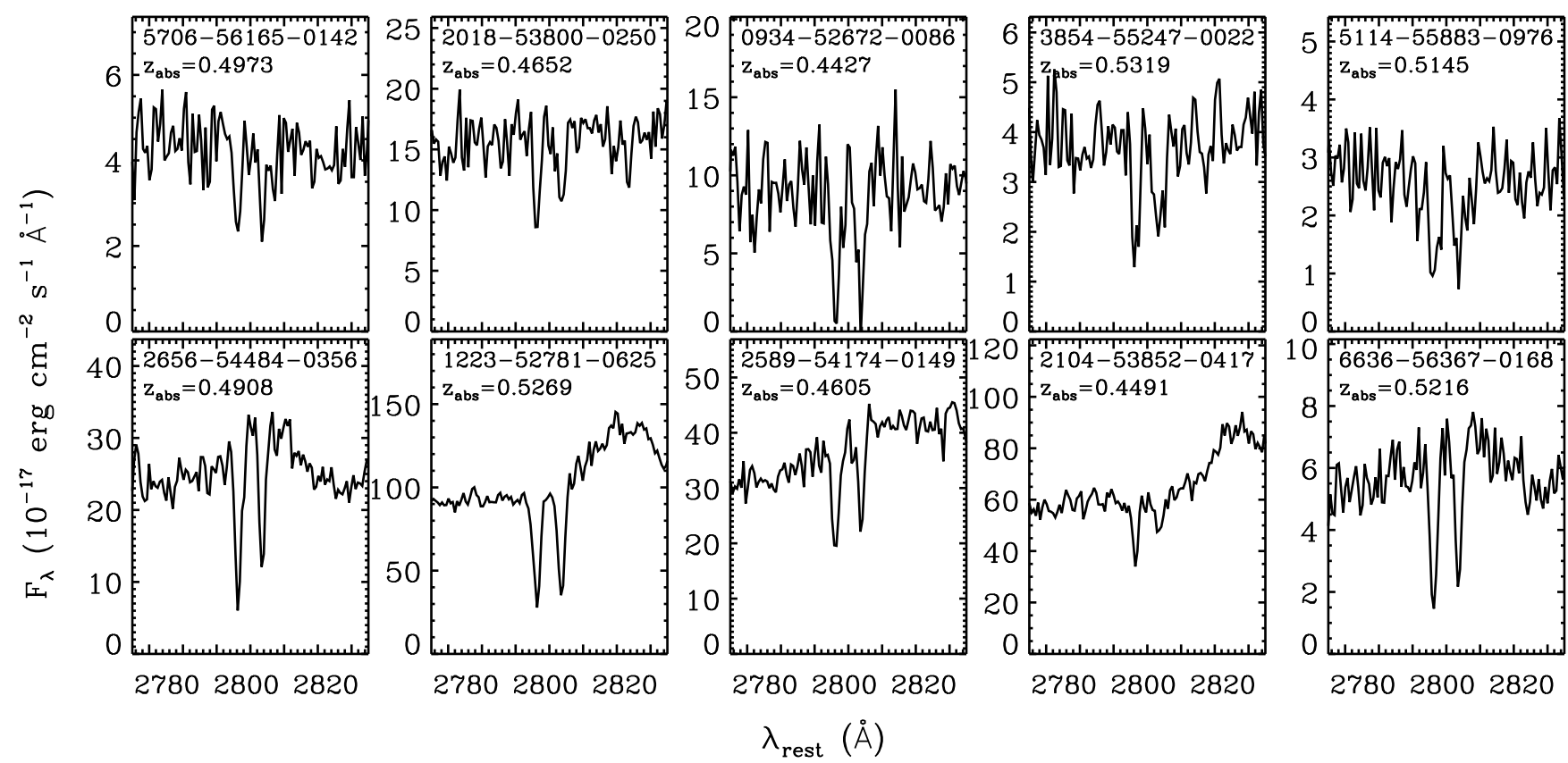

Figure A2. Example spectra for the $\mathrm{Mg}$ II absorbers missed in this study (top). The five cases with the highest S/N of total EW are presented. Spectra of the five absorbers missed in the JHU catalogue (bottom). The spectrum ID and absorber redshift are denoted in each panel.

study to understand why they could not find these absorbers (see also

Zhao et al. 2019).

This paper has been typeset from a $\mathrm{TE}_{\mathrm{E}} / \mathrm{L} \mathrm{T} \mathrm{E} \mathrm{X}$ file prepared by the author. 